\title{
Experimental determination of multiple ionization cross sections in Si by electron impact
}

\author{
Pablo Daniel Pérez, Andrés Sepúlveda, Gustavo Castellano, and Jorge Trincavelli* \\ Instituto de Física Enrique Gaviola and Facultad de Matemática, Astronomía y Física, Universidad Nacional de Córdoba, \\ 5000 Córdoba, Argentina
}

(Received 18 September 2015; published 9 December 2015)

\begin{abstract}
The thin sample method is often used to experimentally determine ionization cross sections, especially when focusing on the low overvoltage region. The simplicity of the formalism involved in this method is very appealing, but some experimental complications arise in the preparation of thin films. In this work, a thick sample method was used to measure the $\mathrm{Si}-K$ x-ray production cross section by electron impact. The good agreement between the results obtained and the values reported in the literature validates the method and the parameters used. The advantages and disadvantages of the method are discussed and its application is extended to the determination of Si multiple-ionization cross sections, where the very low emission rates (around two orders of magnitude lower than the single-ionization case) make the use of the thin sample method impracticable.
\end{abstract}

DOI: 10.1103/PhysRevA.92.062708

PACS number(s): $34.80 . \mathrm{Dp}, 32.30 . \mathrm{Rj}$

\section{INTRODUCTION}

The experimental determination of inner-shell ionization cross sections is a matter of continuous interest in atomic physics [1-8]. As detector performances and speeds are improved, more depurated methodologies can be used to check the increasingly sophisticated and realistic theoretical models used to predict them $[1,5,9]$. The accurate determination of cross sections is of practical application in the field of analytical techniques like electron probe microanalysis, and it is also necessary for the simulation of the electron transport in the scope of materials science and medical physics [10]. Thus, finding a new procedure that may improve or complement the existing ones is of high interest. On the other hand, the measurement of cross sections for low probability phenomena, for example multiple-ionization processes, has deserved the attention and effort of several researchers [11-15], in view of their application to the description of the $\mathrm{x}$-ray emission spectrum. Particularly, the production of satellite lines (arising by either molecular transitions or multiple ionizations) is important in analytical techniques based on x-ray emission: their correct prediction and measurement can be applied to compound speciation and to the validation of molecular orbital theories [16-19].

Traditionally, inner-shell ionization cross sections are measured using thin samples [9,20,21], particularly for high overvoltages. In this situation, the thin sample approximation can be assumed, i.e., the incident electron interacts on average at most once. Thus, the number of characteristic photons $N_{X}\left(E_{o}\right)$ detected when the film is irradiated with electrons of energy $E_{o}$ can be written as

$$
N_{X}\left(E_{o}\right)=\frac{\Delta \Omega}{4 \pi} \epsilon n_{e}\left(E_{o}\right) \frac{N_{A}}{A} \rho t \sigma_{X}\left(E_{o}\right) f,
$$

where $\Delta \Omega$ is the solid angle subtended by the detector window from the electron impact point, $\epsilon$ is the detector intrinsic efficiency at the energy of the detected photon, $n_{e}\left(E_{o}\right)$ is the number of incident electrons, $N_{A}$ the Avogadro's number, $A$ is the atomic weight of the analyzed element, $t$ is the sample

\footnotetext{
*trincavelli@famaf.unc.edu.ar
}

thickness and $\rho$ its mass density, $f$ represents the relative transition probability corresponding to the measured line, and $\sigma_{X}\left(E_{o}\right)$ stands for the X-ray production cross section. It must be noticed that Eq. (1) is only valid for decays to the $K$ shell; for decays to more external shells the situation is more complex because of the subsequent reaccommodation of electrons among the different subshells. This expression is mathematically very simple but the experimental requirements that must be imposed so that the thin-film approximation is fulfilled may be too stringent. To satisfy this approximation, the film must be very thin $(\sim 10 \mathrm{~nm}$ in the case of $\mathrm{Si}$ and other elements with similar atomic number), even when high overvoltages and light elements are under consideration. The preparation of a film of nanometric thickness may become a technological challenge more or less complicated depending on the material; particularly because its thickness must be known with a high degree of precision to lead to precise determinations of ionization cross sections. Another requirement is that the mass density must be homogeneous. These conditions are very difficult to achieve, and special techniques must be used for sample preparation (like magnetron sputtering) and thickness measurement (like Rutherford backscattering spectroscopy). In addition, the film samples are usually deposited on a substrate that can emit characteristic $\mathrm{x}$ rays or bremsstrahlung photons that may excite the film and/or be recorded by the detector, interfering with the analysis. All these issues, along with the low emission rates produced in a thin sample, entail undesired uncertainties that are propagated to the cross-section final result.

On the other hand, multiple ionizations by electron impact are very low probability processes in comparison with simple ionization events. Multiple-ionization satellite lines are caused by light variations in the atomic levels induced by perturbations in the electronic distribution of the atom when it is ionized more than once, e.g., in the case of $K \alpha$ lines, a vacancy in the $K$ shell and another one in the $L$ shell (called spectator hole [22,23]). The different possible decays for a given initial vacancy combined with the several possibilities for the location of the spectator hole originate a complex spectral structure. Double-ionization processes by electron bombardment mainly originate in the ejection of two electrons without any internal rearrangement of bound electrons. These 
events can be classified as shake processes, two-step-one (TS1), and two-step-two (TS2) mechanisms [14]. The shake process is a one-step mechanism that results from a sudden change in the atomic potential due to the electron ejection. The TS1 process can be pictured as if the electron released after the first ionization ejected in turn another bound electron, leaving the atom in a double vacancy state. In the TS2 process the incoming projectile is assumed to interact sequentially with two electrons from the same target atom; thus, TS2 processes are not allowed for incoming photons, which in the energy range of interest are supposed to be absorbed after the interaction.

Bearing in mind the low probability of a multiple-ionization event, the determination of the corresponding cross sections using thin samples would produce excessively high uncertainties and this method would be impracticable. One plausible alternative is the use of a bulk sample, as proposed in Ref. [3]. The thick sample method presents some practical advantages: the sample is easy to prepare, no measurements are required for the sample thickness, photons are registered at a much higher count rate because of the larger interaction volume involved, and there are no substrate contributions.

The main disadvantage of this method is the complexity of the equation that relates $N_{X}\left(E_{o}\right)$ with the ionization cross section. In addition, two assumptions are made in this formalism: the electrons are considered to travel following straight trajectories and emissions due to secondary particles are neglected. Within this degree of approximation, $N_{X}\left(E_{o}\right)$ can be written as

$$
\begin{aligned}
N_{X}\left(E_{o}\right)= & \frac{N_{A}}{A} \frac{\Delta \Omega}{4 \pi} \epsilon n_{e}\left(E_{o}\right) f \int_{0}^{R} \sigma_{X}(E(\rho x)) \\
& \times \exp \left[-\mu_{x} \frac{\cos \alpha}{\cos \beta} \int_{o}^{\rho x} d\left(\rho x^{\prime}\right)\right] d(\rho x),
\end{aligned}
$$

where $R$ is the range for the electrons with energy enough to ionize the level of interest, $\mu_{x}$ is the mass attenuation coefficient for the characteristic $\mathrm{x}$ rays inside the target, and $\alpha$ and $\beta$ are, respectively, the incident and exit angles measured from the sample normal. It is useful to write (5) as a function of the electron stopping power $S(E)$, which is a well-known and tabulated magnitude [24]

$$
S(E)=-\frac{1}{\rho} \frac{d E}{d x}
$$

which can be conveniently replaced in (2), yielding

$$
\begin{aligned}
\frac{N_{X}\left(E_{o}\right)}{n_{e}\left(E_{o}\right)}= & \frac{N_{A}}{A} \frac{\Delta \Omega}{4 \pi} \epsilon f \int_{I}^{E_{o}} \frac{d E}{S(E)} \sigma_{X}(E) \\
& \times \exp \left[-\mu_{X} \frac{\cos \alpha}{\cos \beta} \int_{E}^{E_{o}} \frac{d E^{\prime}}{S\left(E^{\prime}\right)}\right],
\end{aligned}
$$

where $I$ is the ionization threshold energy. Equation (4) can be differentiated with respect to the incident electron energy $E_{o}$ using the Leibniz's rule for differentiation, since the variable involved is present in the integration limits and in the integrand.
The solution for $\sigma_{X}\left(E_{o}\right)$ results in

$$
\begin{aligned}
\sigma_{X}\left(E_{o}\right)= & \frac{A}{N_{A}} \frac{4 \pi}{\Delta \Omega \epsilon f}\left[S\left(E_{o}\right) \frac{d}{d E_{o}}\left(\frac{N_{X}\left(E_{o}\right)}{n_{e}\left(E_{o}\right)}\right)\right. \\
& \left.+\frac{N_{X}\left(E_{o}\right)}{n_{e}\left(E_{o}\right)} \mu_{X} \frac{\cos \alpha}{\cos \beta}\right] .
\end{aligned}
$$

With this expression the cross section $\sigma_{X}\left(E_{o}\right)$ can be obtained from the measurement of $N_{X}\left(E_{o}\right) / n_{e}\left(E_{o}\right)$ at several incident energies $E_{o}$. The downside of this method is that it involves the differentiation of experimental points $N_{X}\left(E_{o}\right)$, with their associated uncertainties. One way to deal with this difficulty is to use the Tikhonov regularization algorithm [3,25]. Nevertheless, in this work, the uncertainties on the photon counting were considerably reduced so that the analytic differentiation of the fitting curve to $N_{X}\left(E_{o}\right) / n_{e}\left(E_{o}\right)$ was sufficiently stable. The number of photons measured at the different $E_{o}$ values, showed a smooth behavior, which justifies the omission of Tikhonov regularization.

It is useful to write Eq. (5) in terms of the number of photons emitted by the sample $P_{X}$, namely

$\sigma_{X}\left(E_{o}\right)=\frac{A}{N_{a}} \frac{1}{f}\left[S\left(E_{o}\right) \frac{d}{d E_{o}}\left(\frac{P_{X}\left(E_{o}\right)}{n_{e}\left(E_{o}\right)}\right)+\frac{P_{X}\left(E_{o}\right)}{n_{e}\left(E_{o}\right)} \mu_{X} \frac{\cos (\alpha)}{\cos (\beta)}\right]$,

where $P_{X}$ can be expressed as

$$
P_{X}=\frac{4 \pi}{\Delta \Omega \epsilon} N_{X}\left(E_{o}\right) .
$$

These formulas are especially useful when the measurements are performed using a wavelength dispersive spectrometer.

In the present research, the thick-sample method was first used to measure the $K$ single-ionization cross section of silicon. This approach was validated by the comparison of the results obtained with the values available in the bibliography. Then, the procedure was extended to the determination of double- and triple-ionization cross sections in Si (ionizations involving simultaneously $K$ and $L$ shell) where the thinsample method is impracticable.

\section{EXPERIMENT}

Spectra were obtained by electron impact on a pure Si bulk standard using an electron microprobe model JEOL 8230. This microprobe involves an energy dispersive spectrometer (EDS) and three wavelength dispersive spectrometer (WDS). The use of a WDS was essential to measure the lines produced by multiple-ionization processes because of the good resolution of this kind of spectrometer. For the present measurements, a pentaerythritol (PET) crystal was used $(2 d=8.742 \AA)$, placed in a Rowland circle with a radius of $140 \mathrm{~mm}$ (Johannson geometry), with a Xe sealed proportional counter attached. The "step" between two successive crystal positions chosen was $30 \mu \mathrm{m}$, according to the criteria explained in Appendix A. Several spectra with different $E_{o}$ were measured, and the crystal position varied from 223.400 to $230.330 \mathrm{~mm}$, covering energies between 1.72457 and $1.77814 \mathrm{keV}$. A normal incident setup was used $\left(\alpha=0^{\circ}\right)$, with an exit angle $\beta=50^{\circ}$. 
TABLE I. Total cumulative time per channel.

\begin{tabular}{lccccc}
\hline \hline $\begin{array}{l}E_{o} \\
(\mathrm{keV})\end{array}$ & $\begin{array}{c}\text { Time } \\
(\mathrm{s} / \text { channel })\end{array}$ & $\begin{array}{c}\text { Total } \\
\text { channels }\end{array}$ & $\begin{array}{l}E_{o} \\
(\mathrm{keV})\end{array}$ & $\begin{array}{c}\text { Time } \\
(\mathrm{s} / \text { channel })\end{array}$ & $\begin{array}{c}\text { Total } \\
\text { channels }\end{array}$ \\
\hline 2.1 & 320 & 146 & 3.5 & 90 & 232 \\
$2.2^{\mathrm{a}}$ & 360 & 146 & 4.0 & 90 & 232 \\
$2.3^{\mathrm{b}}$ & 360 & 146 & 5.0 & 90 & 232 \\
2.4 & 300 & 146 & 6.0 & 75 & 232 \\
2.6 & 300 & 146 & 8.0 & 48 & 232 \\
2.8 & 90 & 232 & 15.0 & 36 & 232 \\
3.0 & 90 & 232 & 20.0 & 36 & 232 \\
\hline \hline
\end{tabular}

${ }^{\text {a }}$ The total time at the $K \alpha L^{1}$ zone was $510 \mathrm{~s} /$ channel.

${ }^{\mathrm{b}}$ The total time at the $K \alpha L^{1}$ zone was $402 \mathrm{~s} / \mathrm{channel}$.

In order to minimize the uncertainties associated with the count number, the collecting time of some measurements was made as large as possible. In fact, the spectra taken at overvoltages very close to 1 were measured during $\sim 12 \mathrm{~h}$ (see Table I). For this reason, the spectra were taken by scanning cumulatively different points of the sample to avoid sample damage, i.e., the sample was irradiated over a point grid and the measuring time of each point was kept below 15 min. Longer exposure times caused significant variations (more than 5\%) in the specimen current. These variations are due to neither beam instabilities nor statistical fluctuations in the detection process, but this effect may occur by sample charging and/or a modification of the physical properties of the sample induced by the electron beam. The total cumulative time per channel is $t_{d} \times(x \times y)$, where $t_{d}$ is the dwell time and $(x \times y)$ is the grid size. For example, the $8-\mathrm{keV}$ spectrum was measured 16 times (a $4 \times 4$ grid) with a dwell time of $3 \mathrm{~s} /$ channel, thus the total cumulative time per channel was $48 \mathrm{~s} /$ channel.

The probe current was measured immediately before and after each point measurement and its stability during the measurement was checked by recording the specimen current.

Some spectral regions required longer measuring times than others, depending on the number of counts registered; for example, the energy range covering the double-ionization zone required more time than the simple ionization zone. In order to reduce uncertainties in these specific ranges, they were measured separately. To obtain the resultant spectrum $I_{\text {sum }}(E)$, a weighted average was performed giving priority to the spectra with larger measuring times. Thus if $I_{a}(E)$ and $I_{b}(E)$ are two spectra with different measuring times per channel (and thereby different statistical uncertainties), the resultant spectrum was calculated as

$$
I_{\text {sum }}(E)=I_{a}(E) \frac{\sqrt{t_{a}}}{\sqrt{t_{a}}+\sqrt{t_{b}}}+I_{b}(E) \frac{\sqrt{t_{b}}}{\sqrt{t_{a}}+\sqrt{t_{b}}},
$$

where $t_{a}$ and $t_{b}$ are the measuring times per channel of spectra $a$ and $b$, respectively. The square roots are introduced so that the weights are the reciprocal of the corresponding statistical uncertainties.

The maximum value of counts per second of the proportional counter was kept below $10^{4}$ counts/s, under the recommendation of the equipment manufacturer, with the purpose of neglecting coincidence losses. Taking this into account, and bearing in mind the dependence of the ionization cross section on the electron energy in the low overvoltage region, spectra corresponding to higher $E_{o}$ values were irradiated with probe currents smaller than those used for low $E_{o}$ spectra: the $20-\mathrm{keV}$ spectrum was measured with a 30-nA current and the $15 \mathrm{keV}$ spectrum, with a 40-nA current, whereas the other spectra were measured with a 100-nA current.

From Eq. (5), it is clear that in order to obtain an absolute value of the ionization cross section, the detector efficiency must be known precisely, because its uncertainties are directly propagated to the cross section final result. The procedure followed to measure the detector efficiency is detailed in Appendix C. The final value used for the effective efficiency in the energy ranges studied is $(2.5 \pm 0.1) \times 10^{-8} \mathrm{keV}$.

In a WDS measurement, high order $x$ rays may distort the spectra, causing errors in the determination of peak intensities, especially in the case of weak transitions. In order to avoid this problem, the electronics of the proportional counter was modified, specifically, the zero and gain parameters of the pulse high analyzer were set to accept a narrow window around the photon energy in such a way that high-order $\mathrm{x}$ rays are not counted.

\section{RESULTS}

An example of the Si spectra obtained is shown in Fig. 1 for $E_{o}=8 \mathrm{keV}$, where different groups of lines are clearly visible. The most intense peaks, corresponding to the single-ionization lines $K \alpha_{1}$ and $K \alpha_{2}$, appear at the low-energy region. They are usually denoted as belonging to the $K \alpha L^{0}$ group, i.e., $K \alpha$ lines with no spectator vacancies in the $L$ shell. On the other hand, at the high-energy side, the $K \alpha L^{1}$ and $K \alpha L^{2}$ groups can be seen, which are respectively caused by double ionization (one spectator hole in the $L$ shell) and triple ionization (two spectator holes in the $L$-shell). According to Cauchois et al. [26], the double-ionization lines $K \alpha^{\prime}, K \alpha_{3}$, and $K \alpha_{4}$ are

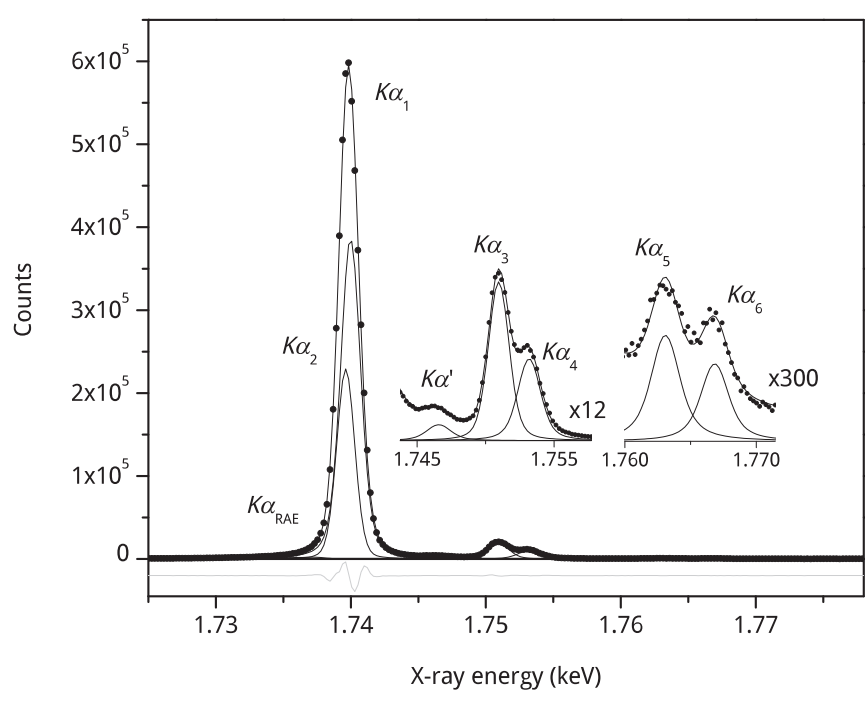

FIG. 1. Si spectrum obtained for $8-\mathrm{keV}$ electron impact. Dots: experimental data; black solid line: spectral fitting; grey solid lines: contributions of each peak. The insets show double- and triple-ionization satellite zoomed regions, keeping the abscissa scale unaltered. 
located at $7.79,11.3$, and $13.2 \mathrm{eV}$, respectively, relative to the main peak $K \alpha_{1,2}$, and the triple ionization $K \alpha_{5}$ and $K \alpha_{6}$ are shifted in 23.8 and $26.3 \mathrm{eV}$, respectively. These values are in good agreement with the peaks measured at the higher energy side of the main peak, displaced from it $6.7 \pm 0.2$, $11.10 \pm 0.05,13.32 \pm 0.04,23.2 \pm 0.4$, and $26.9 \pm 0.5 \mathrm{eV}$. The four latter values can be unequivocally identified with $K \alpha_{3}, K \alpha_{4}, K \alpha_{5}$, and $K \alpha_{6}$, respectively; in the case of $K \alpha^{\prime}$, there is a difference between the results obtained in this work and [26], although in a more recent investigation based on proton excitation [23], a $K \alpha^{\prime}$ shift of $6.57 \pm 0.03$ was found, which is in agreement with our outcome. In addition, a structure probably caused by a $K L M$ radiative Auger emission (RAE) was also identified in the $K \alpha L^{0}$ group (see Fig. 1) [2].

Spectra were fitted using a parameter optimization code [27] which minimizes the quadratic differences between the experimental data and an analytical model for the spectrum. This model takes into account several physical phenomena such as bremsstrahlung, characteristic peak generation, photon attenuation in the sample, instrumental broadening and efficiency, etc. In view of the closeness between $K \alpha_{1}$ and $K \alpha_{2}$ lines, they were specially treated, because the spectrometer cannot resolve them completely. Thus, some parameters like natural linewidth, energy, and transition probability are very correlated. To avoid this obstacle, a strategy based on keeping a constant ratio between $K \alpha_{1}$ and $K \alpha_{2}$ intensities was followed [2,19]. A Voigt profile [28] was used for every line except for the RAE structure, for which a convolution between the expression given by Enkisch [29] and a Gaussian profile was used.

The number of emitted photons $P_{X}$ in Eq. (6) was obtained from the deconvoluted peak area $N_{X}^{\prime}$, as explained in Appendixes B and C. The values for electron stopping power and mass attenuation coefficients were taken from the ESTAR and XCOM platforms [30,31].

\section{A. Single-ionization $x$-ray production cross section}

The results obtained for $N_{x}^{\prime}$ corresponding to singleionization decays ( $K \alpha_{1,2}$ doublet) at several incidence energies $E_{o}$ are plotted in Fig. 2. It must be noted that the points describe a very smooth curve due to the low statistical error obtained by carrying out long time measurements as mentioned in Sec. II. This fact leads to reliable results without the need of regularization methods.

In order to perform the differentiation involved in Eq. (6), an analytical expression for $N_{X}^{\prime}\left(E_{o}\right)$ was fitted:

$$
N_{X}^{\prime}\left(E_{o}\right)=e^{\left[c_{1}\left(E_{o}-c_{2}\right)^{-c_{3}}\right]}\left(c_{4} E_{o}^{-c_{5}}-c_{6}\right),
$$

where the $c_{i}$ 's are parameters obtained from least-squares fitting. This function along with its derivative was used in Eq. (6) to obtain the X-ray production cross section shown in Fig 3.

The uncertainty of the area $N_{X}^{\prime}\left(E_{o}\right)$ was assessed by propagating the expression (B1), considering that the error in $I(E)$ is estimated as $\sqrt{I(E)}$. On the other hand, an upper bound for the uncertainty in the derivative involved in Eq. (6) was estimated by calculating the derivative as an incremental ratio between two consecutive experimental values of $N_{X}^{\prime}\left(E_{o}\right)$ and propagating the involved uncertainties. The probe current

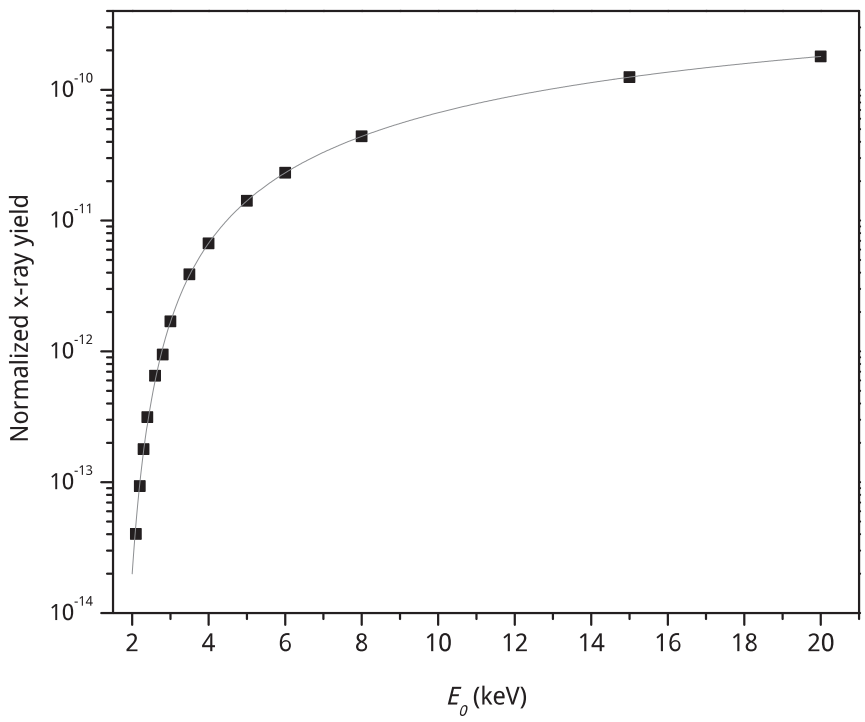

FIG. 2. Peak area $N_{X}^{\prime}$ for the main peak $K \alpha L^{0}$ (single ionization) as a function of the incident energy $E_{o}$. Dots: experimental data; solid line: analytical fit. The error bars are smaller than the point size.

variation immediately before and after each spectrum acquisition was below $0.05 \%$ in all measurements; this variation was considered as the uncertainty in the number of incident electrons $n_{e}\left(E_{o}\right)$. The error estimates for the stopping power values were close to $10 \%$ in the analyzed region [32]. In the case of the attenuation coefficient, the uncertainty was also around $10 \%$, because it is very close to the absorption threshold [33]. For the relative transition probability $f$, its value was taken as $0.967 \pm 0.005$, considering the results given in [2,34]. The uncertainties associated with the angles $\alpha$ and $\beta$ were found to be negligible as compared to the other values. Finally, the uncertainties of the $\mathrm{x}$-ray production cross section values were obtained by propagating errors in Eq. (6).

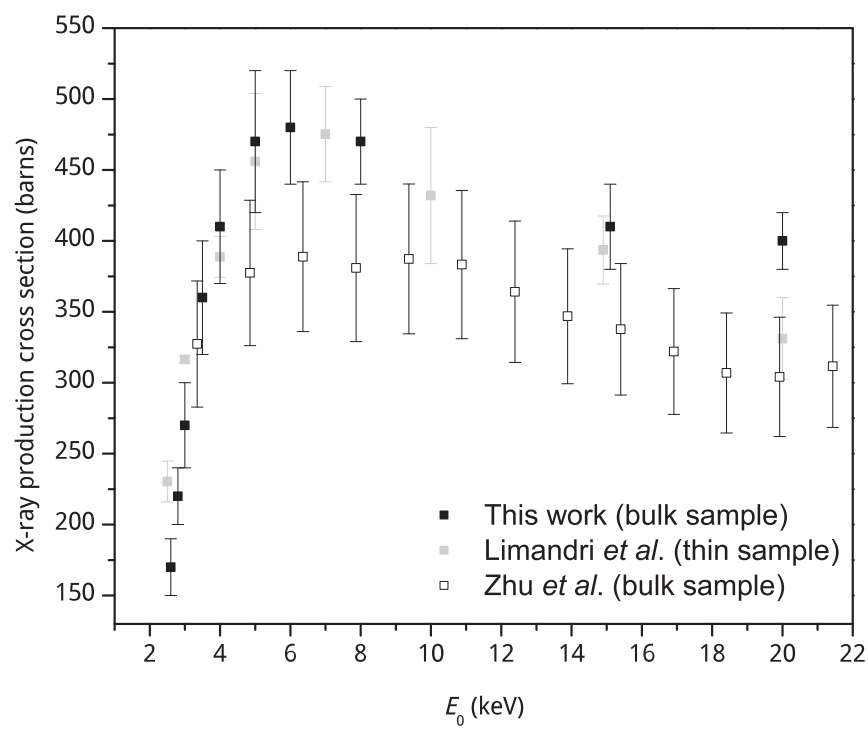

FIG. 3. Silicon $K$-shell x-ray production cross section for single ionization. Black dots: present results; white dots: Zhu et al. [35]; gray dots: Limandri et al. [1] 
Figure 3 displays the results obtained for $K$-shell single ionization, along with data obtained by means of the bulk sample method [35] and through measurements performed irradiating a thin target [1]. It can be seen that there is a good agreement between the $\mathrm{x}$-ray production cross section values obtained here and the other experimental results, particularly with those from Ref. [1]. The differences between the present data and those from [35] could be due to the fact that their results were obtained using an EDS instead of a WDS. The reasonable performance achieved validates the method followed in the present work, i.e., the spectrometer efficiency obtained, the peak integration carried out, the expression fitted for $N_{x}^{\prime}\left(E_{o}\right)$, the spectral deconvolution and the expression (6) can all be used consistently.

The good results obtained for silicon single-ionization cross section allowed us to safely extend the thick sample procedure to the determination of multiple-ionization cross sections, where no experimental alternative is available.

\section{B. X-ray production cross sections by multiple ionizations}

The very low probabilities related to multiple-ionization events inhibit the use of thin samples to determine multipleionization cross sections. The thick sample approach is instead quite suitable for the present purpose. In order to determine the double-ionization $\mathrm{x}$-ray production cross section $\sigma_{X}\left(K \alpha L^{1}\right)$, the $K \alpha L^{1}$ group was considered. The result obtained can be associated with the probability of producing one vacancy in the $K$ shell and one in the $L$ shell, no matter which specific $L$ subshell is involved. Analogously, the $K \alpha L^{2}$ group was considered to obtain the x-ray production cross section $\sigma_{X}\left(K \alpha L^{2}\right)$ for triple ionization, i.e., the probability of producing one vacancy in the $K$ shell and two (any) spectator holes in the $L$ shell, normalized by the number of atoms per unit area. The peak areas $N_{x}^{\prime}\left(K \alpha L^{1}\right)$ and $N_{x}^{\prime}\left(K \alpha L^{2}\right)$ obtained for the $K \alpha L^{1}$ and $K \alpha L^{2}$ groups at several incident energies are plotted in Fig. 4.

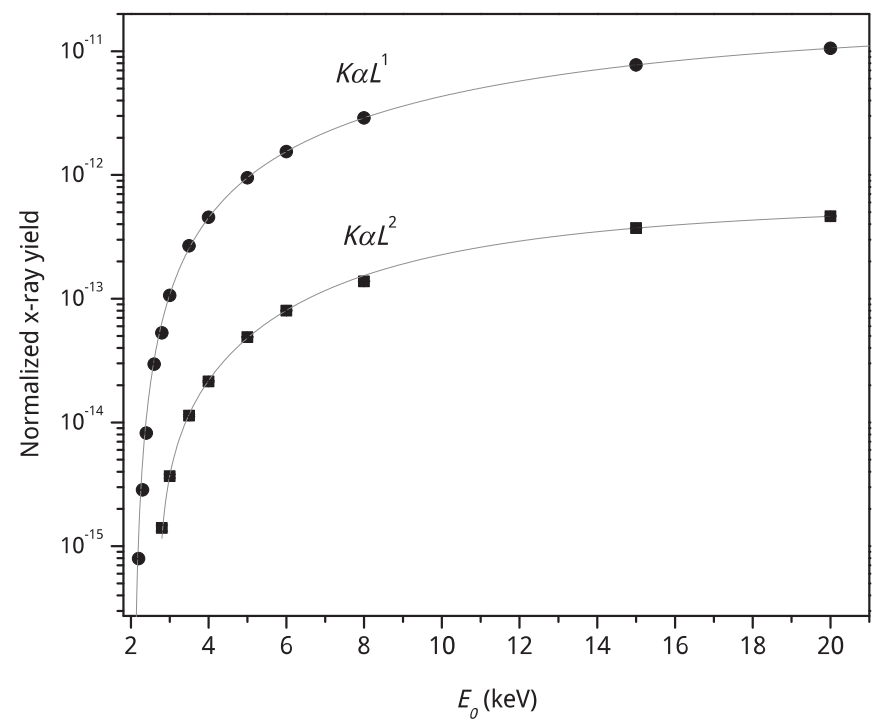

FIG. 4. Peak area $N_{X}^{\prime}$ as a function of the incident energy $E_{o}$. Solid circles: $K \alpha L^{1}$ group (double ionization); solid squares: $K \alpha L^{2}$ (triple ionization). The error bars are smaller than the point size.

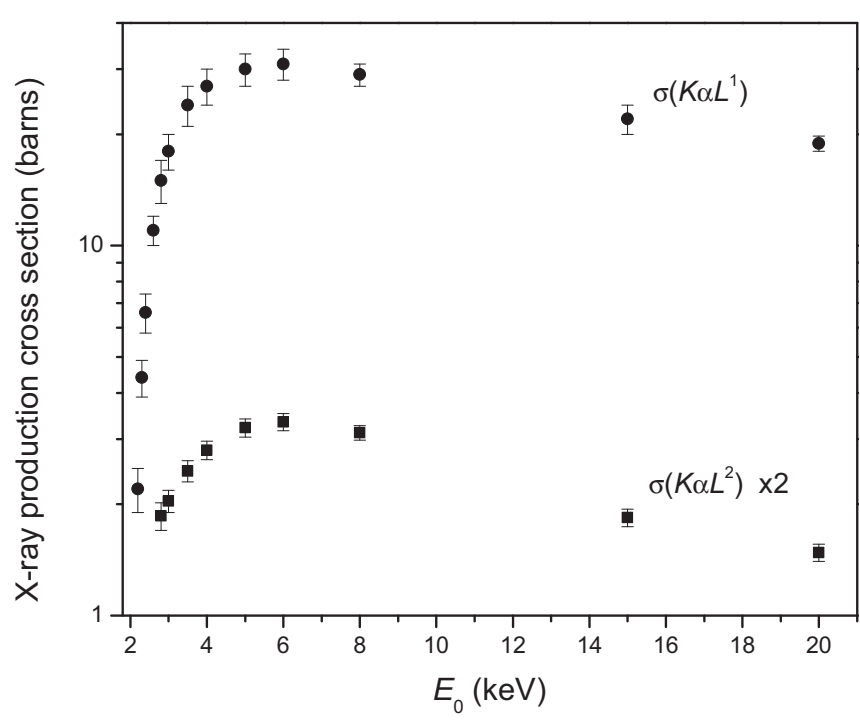

FIG. 5. Silicon x-ray production cross section for $K$ lines with one $L$ spectator hole (solid circles) and with two $L$ spectator holes (solid squares) as a function of the incident energy $E_{o}$.

These data were also fitted using the functional behavior given in Eq. (8), and the resulting function was replaced in Eq. (6) along with its derivative. The values obtained for the multiple-ionization x-ray production cross sections are shown in Fig. 5 and detailed in Table II. These values were not compared to other results because the only quantitative information for multiple-ionization cross sections by electron impact available in the literature is restricted to satellite peak intensities relative to a reference value, for example the main peak intensity, and they are not corrected by self-absorption or electron energy loss, like the data published here.

The trend followed by $\sigma_{X}\left(K \alpha L^{0}\right), \sigma_{X}\left(K \alpha L^{1}\right)$, and $\sigma_{X}\left(K \alpha L^{2}\right)$ are very similar. In all cases, the maximum probability of ionization (single or multiple) is reached at energies between 6 and $7 \mathrm{keV}$. Remarkably, $\sigma_{X}\left(K \alpha L^{2}\right)$ shows

TABLE II. Silicon $\mathrm{x}$-ray production cross sections for single, double and triple ionization obtained by means of the thick sample method.

\begin{tabular}{lccc}
\hline \hline $\begin{array}{l}E_{o} \\
(\mathrm{keV})\end{array}$ & $\begin{array}{c}\sigma_{X}\left(K \alpha L^{0}\right) \\
(\text { barns })\end{array}$ & $\begin{array}{c}\sigma_{X}\left(K \alpha L^{1}\right) \\
\text { (barns) }\end{array}$ & $\begin{array}{c}\sigma_{X}\left(K \alpha L^{2}\right) \\
\text { (barns) }\end{array}$ \\
\hline 2.1 & $36 \pm 4$ & & \\
2.2 & $58 \pm 6$ & $2.2 \pm 0.3$ & \\
2.3 & $83 \pm 9$ & $4.4 \pm 0.5$ & \\
2.4 & $110 \pm 10$ & $6.6 \pm 0.8$ & \\
2.6 & $170 \pm 20$ & $11 \pm 1$ & \\
2.8 & $220 \pm 20$ & $15 \pm 2$ & $0.93 \pm 0.08$ \\
3.0 & $270 \pm 30$ & $18 \pm 2$ & $1.02 \pm 0.07$ \\
3.5 & $360 \pm 40$ & $24 \pm 3$ & $1.23 \pm 0.08$ \\
4.0 & $410 \pm 40$ & $27 \pm 3$ & $1.40 \pm 0.08$ \\
5.0 & $470 \pm 50$ & $30 \pm 3$ & $1.61 \pm 0.09$ \\
6.0 & $480 \pm 40$ & $31 \pm 3$ & $1.67 \pm 0.09$ \\
8.0 & $470 \pm 30$ & $29 \pm 2$ & $1.56 \pm 0.07$ \\
15.0 & $410 \pm 30$ & $22 \pm 2$ & $0.92 \pm 0.05$ \\
20.0 & $400 \pm 20$ & $18.9 \pm 0.9$ & $0.74 \pm 0.04$ \\
\hline \hline
\end{tabular}


a pronounced decrease from the maximum at $6 \mathrm{keV}$, above $20 \mathrm{keV}$ bearing values smaller than for low energies.

As mentioned in the Introduction, TS2 processes are not allowed for incoming photons. Measurements using photon beams may allow us to establish a relationship between the total $K L$ double-ionization cross section and the particular contribution due to TS2 processes. According to Mauron et al. [14] this ratio is proportional to $Z$, which, using the results obtained here for silicon, would correspond to a TS2 contribution between $35 \%$ and $40 \%$. Further experiments would be necessary to corroborate this issue.

The bulk sample approach involved in this work allows us to accurately assign the electron energy for which the cross sections are assessed, avoiding the need of defining effective energies, as in [14]. Despite this advantage, it was not possible to observe the oscillations reported by Mauron et al. in $K \alpha_{3}$ and $K \alpha_{4}$ yields; instead, a monotonic increase with the incident energy was observed for them.

\section{CONCLUSION}

The absolute $K$-shell x-ray production cross section was experimentally determined for $\mathrm{Si}$, in the energy range 2.1$20 \mathrm{keV}$, following a bulk sample method. The reliability of the whole procedure was evidenced by the good agreement between the results obtained here for single ionization and the ones available in the literature. This agreement demonstrates that the cross section values were produced through Eq. (6) using a consistent procedure, by combining the detector efficiency curve obtained, the long time measurements carried out to reduce uncertainties, the proper deconvolution of peak intensities and the analytical curves fitted to them. Regularization methods were not necessary in the special case studied here (silicon at overvoltages between 1 and 10), because the uncertainties were minimized.

It can be stated that the thin and thick sample methods are complementary: for high overvoltages, the former approximation is more reliable than the electron straight trajectories assumed in the latter; on the other hand, the thick sample method is more suitable for low overvoltages, where the thin-film hypothesis is not easily satisfied and the inherent low statistics hampers the experimental determinations.

The thick sample method was extended to the determination of double- and triple-ionization cross sections. It must be emphasized that these cross sections cannot be determined by using a thin sample, due to the low probabilities involved.

The procedure described here may be applied to similar situations where very low probability events are involved. This is, for instance, the case of the determination of x-ray production cross sections associated with hypersatellite lines.

\section{ACKNOWLEDGMENTS}

The authors acknowledge the Laboratorio de Microscopía Electrónica y Microanálisis (LAMARX) of the Universidad Nacional de Córdoba, where all the spectra used in this work were measured.

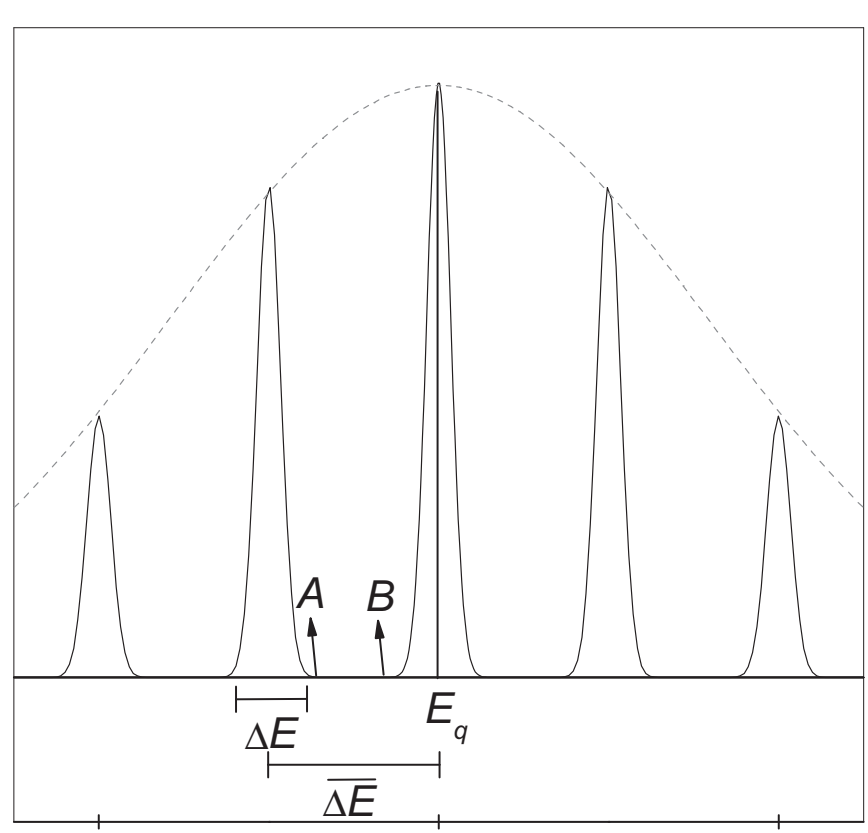

FIG. 6. Scheme depicting the $\Delta E$ and $\overline{\Delta E}$ parameters.

\section{APPENDIX A: CHOOSING THE STEP LENGTH $\overline{\Delta E}$}

Let $I(E)$ be the equipment output that gives the number of photons detected between $E$ and $E+\Delta E$, where $\Delta E$ is related to the spectrometer resolution, and let $\overline{\Delta E}$ be the energy difference between two successive positions of the analyzing crystal (see Fig. 6). In an energy dispersive spectrometer (EDS) $\overline{\Delta E}$ is equal to $\Delta E$, thus the intensity yield of a peak can be calculated just by adding the intensities of all channels involved in the peak. In the case of a WDS, the intervals are different, so that the total intensity of a measured peak cannot be obtained in the same way. If $\Delta E \geqslant \overline{\Delta E}$, count overlap would distort the characteristic intensity recorded. On the other hand, if $\Delta E \leqslant \overline{\Delta E}$ there would be photons not taken into account (between points $\mathrm{A}$ and $\mathrm{B}$ in Fig. 6, for example). The latter condition is anyway preferred, and care must be taken in order to properly count the photons entering the detector.

Several 8-keV Si- $K \alpha$ spectra were measured with different step lengths $\overline{\Delta E}$ of the analyzing crystal, from 15 to $90 \mu \mathrm{m}$, to check if the condition $\Delta E \leqslant \overline{\Delta E}$ is satisfied. All the obtained spectra follow approximately the same curve, as can be seen in Fig. 7, which demonstrates that even with the $15-\mu \mathrm{m}$ step length, the measurement is in a nonoverlapping regime. A compromise configuration for reasonable measuring time and good statistics was found for $\overline{\Delta E}=30 \mu \mathrm{m}$.

\section{APPENDIX B: PEAK INTEGRATION}

As mentioned in the previous Appendix, special care has to be taken when obtaining the photon yield of a peak measured with a WDS because its channels are not immediately adjacent. In the case of an EDS measurement the peak intensity yield is calculated by adding the intensities of the channels involved in the peak. However in the case of a WDS measurement, this sum varies with the particular selection of the $\overline{\Delta E}$ value chosen for the measurement, i.e., the intensity yield of a peak cannot 


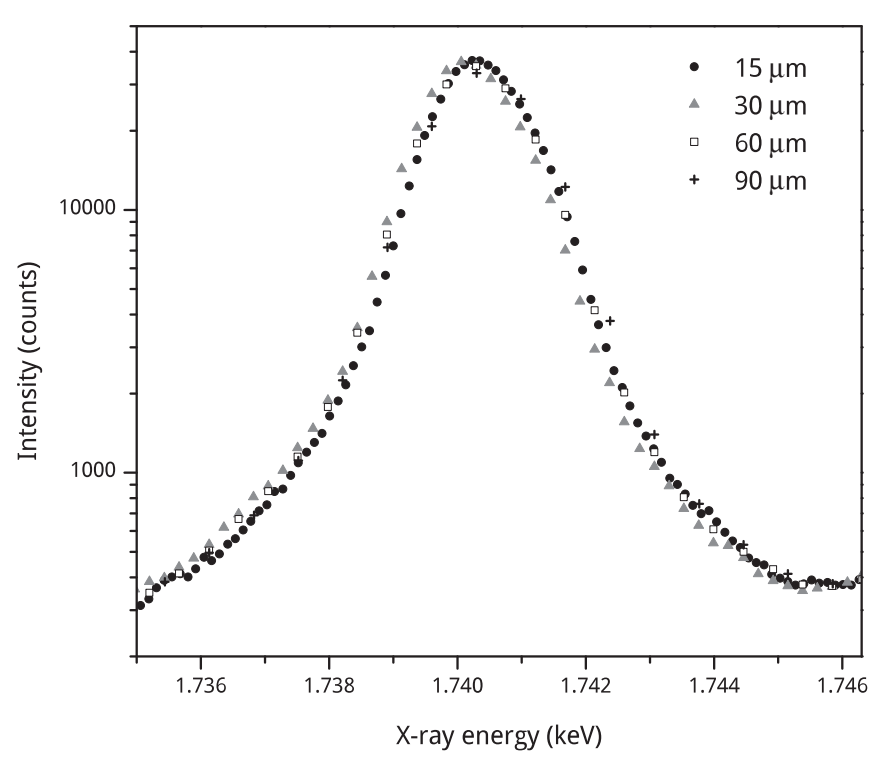

FIG. 7. Silicon $K \alpha_{1,2}$ spectra obtained with $8-\mathrm{keV}$ electrons and different step lengths for the PET analyzing crystal.

be obtained by a simple sum of the intensities registered in the corresponding channels.

On the other hand, the area of a peak located at the energy $E_{q}$ corresponding to the $q$ peak does not depend on the particular election of $\overline{\Delta E}$, as it can be seen from Fig. 7. Then, the peak area $N_{X}^{\prime}$ can be defined as the area under the curve followed by the experimental points:

$$
N_{X}^{\prime}=\int_{E_{q}-w}^{E_{q}+w} I(E) d E,
$$

where $w$ is an energy such that the interval between $E_{q}-w$ and $E_{q}+w$ covers all the photons emitted by the transition considered. It is clear that $N_{X}^{\prime}$ has units of energy and is proportional to the total number of characteristic photons $P_{q}$ emitted by the target.

The data shown in Figs. 2 and 4 were calculated using the expression (B1), $I(E)$ being the intensity of the deconvoluted peaks and $w=10 \mathrm{eV}$.

\section{APPENDIX C: EFFECTIVE EFFICIENCY}

The WDS efficiency depends on parameters that are too complicated to be determined individually; for instance, the analyzing crystal reflectivity, the transmittance of the proportional counter sealant window, the proportional counter efficiency itself, etc. Thus, the derivation of an analytical expression based on these parameters is not practical for the objectives of this work. On the other hand, a WDS effective efficiency curve that takes into account all these factors can be determined experimentally.

In this work, the effective efficiency $\varepsilon$ was defined as the ratio between the peak area $N_{X}^{\prime}$ (see Appendix B) normalized by the number of incident electrons $n_{e}$, and the number of characteristic photons $P_{q}$ emitted by the sample, namely

$$
\varepsilon\left(E_{q}\right)=\frac{N_{X}^{\prime} / n_{e}}{P_{q}} .
$$

It has to be noted that the efficiency defined in this way has energy units, because the peak area $N_{X}^{\prime}$ has energy units.

According to Eq. (C1), $P_{q}$ has to be known to obtain $\varepsilon\left(E_{q}\right)$. This can be achieved by using an EDS spectrometer whose efficiency has been previously well established. To this aim, the following relationship between $P_{q}$ and the number of characteristic photons $I_{\mathrm{EDS}}\left(E_{i}\right)$ measured by the EDS at energy $E_{i}$ can be written

$$
P_{q}=\sum_{i} \frac{I_{\mathrm{EDS}}\left(E_{i}\right)}{\frac{\Delta \Omega}{4 \pi} \varepsilon_{\mathrm{int}}\left(E_{i}\right) n},
$$

where $\Delta \Omega / 4 \pi$ is the solid angle subtended by the detector window from the impact point of the electrons on the sample, $\varepsilon_{\text {int }}\left(E_{i}\right)$ is the intrinsic efficiency at energy $E_{i}, n$ is the number of incident electrons for the EDS measurement, and the sum includes all the channels $i$ within the peak. Thus

$$
\varepsilon\left(E_{q}\right)=\frac{N_{X}^{\prime}}{n_{e}}\left(\sum_{i} \frac{I_{\mathrm{EDS}}\left(E_{i}\right)}{\frac{\Delta \Omega}{4 \pi} \varepsilon_{\mathrm{int}}\left(E_{i}\right) n}\right)^{-1} .
$$

The procedure followed to determine the solid angle and the intrinsic efficiency of the EDS was explained elsewhere [1]. The solid angle obtained was $(1.15 \pm 0.05) \times 10^{-4} \mathrm{sr}$, whereas $\varepsilon_{\text {int }}$ was evaluated at $1.74 \mathrm{keV}$, resulting in $0.707 \pm 0.007$.

In order to find $\varepsilon\left(E_{\mathrm{Si}-\mathrm{K} \alpha}\right)$, a $K \alpha$ spectrum of $\mathrm{Si}$ was measured using 8-keV electrons and both EDS and WDS detectors. Using Eq. (C3), the efficiency in the $K \alpha$ region of the PET-WDS was determined to be $(2.5 \pm 0.1) \times 10^{-8} \mathrm{keV}$. With this value and $N_{X}^{\prime}$ as explained in Appendix B, the net number of photons emitted by the sample $P_{X}$ involved in Eq. (6) can be obtained from

$$
P_{X}=\frac{N_{X}^{\prime}}{\varepsilon\left(E_{\mathrm{Si}-\mathrm{K} \alpha}\right)} .
$$

[1] S. P. Limandri, M. A. Z. Vasconcellos, R. Hinrichs, and J. C. Trincavelli, Phys. Rev. A 86, 042701 (2012).

[2] S. P. Limandri, R. D. Bonetto, A. C. Carreras, and J. C. Trincavelli, Phys. Rev. A 82, 032505 (2010).

[3] Z. An and Q. Hou, Phys. Rev. A 77, 042702 (2008).

[4] S. F. Barros, V. R. Vanin, N. L. Maidana, and J. M. FernándezVarea, J. Phys. B 48, 175201 (2015).

[5] Y.-K. Kim and P. M. Stone, J. Phys. B 40, 1597 (2007).
[6] I. Török, T. Papp, and S. Raman, Nucl. Instrum. Methods Phys. Res., Sect. B 150, 8 (1999).

[7] J. M. Fernández-Varea, V. Jahnke, N. L. Maidana, A. A. Malafronte, and V. R. Vanin, J. Phys. B 47, 155201 (2014).

[8] J. P. Santos, F. Parente, and Y.-K. Kim, J. Phys. B 36, 4211 (2003).

[9] X. Llovet, C. J. Powell, F. Salvat, and A. Jablonski, J. Phys. Chem. Ref. Data 43, 013102 (2014). 
[10] F. Salvat, J. M. Fernández-Varea, and J. Sempau, PENELOPE2006: A Code System for Monte Carlo Simulation of Electron and Photon Transport (OECD Publications, Paris, 2006).

[11] J. Hoszowska, J.-C. Dousse, J. Szlachetko, Y. Kayser, W. Cao, P. Jagodziński, M. Kavčič, and S. H. Nowak, Phys. Rev. Lett. 107, 053001 (2011).

[12] M. Kavčič, A. Karydas, and C. Zarkadas, Nucl. Instrum. Methods Phys. Res., Sect. B 222, 601 (2004).

[13] M. Kavčič, J.-C. Dousse, J. Szlachetko, and W. Cao, Nucl. Instrum. Methods Phys. Res., Sect. B 260, 642 (2007).

[14] O. Mauron and J.-C. Dousse, Phys. Rev. A 66, 042713 (2002).

[15] K. Fennane, J.-C. Dousse, J. Hoszowska, M. Berset, W. Cao, Y.-P. Maillard, J. Szlachetko, M. Szlachetko, and M. Kavčič, Phys. Rev. A 79, 032708 (2009).

[16] M. T. Deluigi and J. Riveros, Chem. Phys. 325, 472 (2006).

[17] S. Limandri, S. Ceppi, G. Tirao, G. Stutz, C. Sánchez, and J. Riveros, Chem. Phys. 367, 93 (2010).

[18] M. C. Rowe, A. J. Kent, and R. L. Nielsen, Chem. Geol. 236, 303 (2007).

[19] P. D. Pérez, A. C. Carreras, and J. C. Trincavelli, J. Phys. B 45, 025004 (2012).

[20] C. S. Campos, M. A. Z. Vasconcellos, X. Llovet, and F. Salvat, Phys. Rev. A 66, 012719 (2002).

[21] A. Sepúlveda, A. P. Bertol, M. A. Z. Vasconcellos, J. Trincavelli, R. Hinrichs, and G. Castellano, J. Phys. B 47, 215006 (2014).

[22] T. Åberg, Phys. Rev. 156, 35 (1967).

[23] M. Kavčič, Phys. Rev. A 68, 022713 (2003).

[24] H. Paul and A. Schinner, Nucl. Instrum. Methods Phys. Res., Sect. B 227, 461 (2005).

[25] J. Weese, Comput. Phys. Commun. 69, 99 (1992).
[26] Y. Cauchois and C. Sénémaud, Tables of Wavelengths of X-ray Emission Lines and Absorption Edges (International Tables of Selected Constants 18) (Pergamon, Oxford, 1978).

[27] S. P. Limandri, J. C. Trincavelli, R. D. Bonetto, and A. C. Carreras, Phys. Rev. A 78, 022518 (2008).

[28] S. P. Limandri, R. D. Bonetto, H. O. D. Rocco, and J. C. Trincavelli, Spectrochim. Acta, Part B 63, 962 (2008).

[29] H. Enkisch, C. Sternemann, M. Paulus, M. Volmer, and W. Schülke, Phys. Rev. A 70, 022508 (2004).

[30] M. Berger, J. Coursey, M. Zucker, and J. Chang, ESTAR, PSTAR, and ASTAR: Computer Programs for Calculating Stopping-Power and Range Tables for Electrons, Protons, and Helium Ions (version 1.2.3), http://physics.nist.gov/Star (2005) (online; accessed Sep. 2014).

[31] M. J. Berger, J. Hubbell, S. Seltzer, J. Chang, J. Coursey, R. Sukumar, D. Zucker, and K. Olsen, XCOM: Photon Cross Section Database (version 1.5), http://physics.nist.gov/xcom (2010) (online; accessed Sep. 2014).

[32] M. Berger, J. Coursey, M. Zucker, and J. Chang, ESTAR, PSTAR, and ASTAR: Computer Programs for Calculating Stopping-Power and Range Tables for Electrons, Protons, and Helium Ions (version 1.2.3), http://physics.nist.gov/ PhysRefData/Star/Text/method.html (2005) (online; accessed Sep. 2014).

[33] C. T. Chantler, J. Phys. Chem. Ref. Data 24, 71 (1995).

[34] S. I. Salem, T. H. Falconer, and R. W. Winchell, Phys. Rev. A 6, 2147 (1972).

[35] J. Zhu, Z. An, M. Liu, and L. Tian, Phys. Rev. A 79, 052710 (2009). 